\title{
Prosodic and Gestural Cues for Navigations around Mental Space
}

\author{
JUNE LUCHJENBROERS \\ University of Wales at Bangor
}

\section{Introduction}

This paper reports on research that explores how lexical, prosodic, and gestural information combine to provide discourse participants with the appropriate cues needed to set up and structure mental spaces. ${ }^{1}$ The particular issues considered here include: (i) the role of prosodic stress in alerting hearers to the necessary movements around conceptual space, (ii) the role of gestural information in navigating conceptual space, and (iii) the extent to which prosodic and gestural cues converge to help the hearer to navigate the speaker's use of mental space. This paper builds on earlier work that has used information flow categories as a predictor of attentional focus in mental space construction and navigation. This research agenda now includes audio and video data of negotiated talk, which has raised important, new questions about the primacy of information flow categories as predictors of mental space activity and about the facilitation of the discourse building process by prosodic and/or gestural information.

\section{The Data}

\subsection{Research Design}

The data used for this paper comes from a larger videotaped study into negotiated talk between one Australian male or female student, discussing behaviors to be avoided at university, and either one other Australian or foreign student. A total of 32 interactional dyads was collected.

Subjects were recorded in a soundproof room positioned diagonally opposite each other. The purpose of this positioning was to maximize the view for the analyst (sitting in the next room behind a large tinted window) and the video recorder without drawing undue attention to either. Subjects reported that they found the analyst easy to ignore, but that was less true of the video recorder.

\footnotetext{
${ }^{1}$ The research drawn upon in this paper was supported by a postdoctoral grant (supplemented by grant \#122 $336004000 \mathrm{R}$ CLTR/UQNSRG088G 97) to the author with the University of Queensland (Australia). I thank Eve Sweetser, Satoro Kita, and Susan Duncan for their feedback at the presentation, and Wilbert Kraan for running his eye over the draft manuscript. All errors are mine.
} 
June Luchjenbroers

The participants in each dyad were given the task of devising guidelines (to be given to faculty) about how new students should avoid the pitfalls associated with (a) cheating, or (b) plagiarism. Each dyad lasted roughly 30 minutes.

Subjects were drawn from any discipline and at differing levels of study (i.e. both undergraduate and postgraduate students were included). Participants had either not previously met or did not know each other well, and they were paid for their participation.

\subsection{Initial Observations}

This paper focuses on 12 of the 32 interactional dyads, which is the total number of dyads involving only Australian students. The Australians-only data is made up of 4 male-male dyads, 4 male-female dyads, and 4 female-female dyads.

The first discovery made in these data is that there are remarkable individual and gender differences in how much subjects make use of gesture during conversation. Australian women gesture far more than Australian men, and noticeably more so when talking to another Australian woman than when talking to an Australian man. Australian men gesture very little, and particularly so when talking to another Australian man.

I have therefore found it more practical to first find good gesture examples and establish how lexical and prosodic cues fit in, and then to start at the beginning of a conversation and see how the chosen factors converge. In general, the examples given in this paper were drawn from female-female dyads. In contrast, analysis of prosodic information can easily start at the beginning of talk because lexical and prosodic information go hand in hand.

\section{2. $\quad$ Prosodic Stress and Focus}

The chosen prosodic feature for this project is stress assignment, and the research objective is to determine how stress conveys mental space information. Like others in the discourse field, I view discourse as a process of mutual ground construction where speakers are responsible for giving addressees adequate cues to derive their intended meanings, and addressees are responsible for making a determined search for those meanings. This discourse building process has been largely mapped using the range of functionalist tools at the analyst's disposal: focus, topic, newness, and givenness (cf. Chafe 1994, Lambrecht 1994, Luchjenbroers 1993). However, notable difficulties exist with the definitions of these tools and with adequately defining the relationships between them (Luchjenbroers in press, in progress).

In particular, prosodic stress most closely associates with the notion of focus; however, focus, like many other concepts in the discourse analyst's armory, is vague and often misused. Discourse elements may achieve focus in the mind of the hearer, and those same elements may be stressed in the speaker's attempt to give hearers adequate cues about speaker-intended meanings, but the two processes are not the same. In essence, what happens in the speaker's mind ought not to be confused with what is thought to happen in the hearer's mind. In my work, 
stress assignment is a hearer-oriented, speaker strategy, and focus is a property of the hearer's cognitive system.

The second major association for stress is with the information flow category of new/newsworthy information. I have found this to be a largely reliable association in that new/newsworthy information tends to attract increased stress in the speaker's output; however, information flow, like focus, is also a property of the hearer's cognitive system.

In effect, speakers have control over lexical choice, grammatical placements, and prosody, but the key conceptual processes with which these linguistic strategies are generally associated (focus and information flow) belong to the hearer's cognitive system. The communication process relies on speakers using those cues within their control (e.g. stress and lexical placement) to enable the hearer to follow the speaker-intended meaning. This involves speakers making assumptions about the likely information flow status that information would have in the mind of the hearer in order for them to adequately structure their outputs-hence, the discourse analysts' tendency to collapse speaker stimuli with expected hearer cognitive phenomena. However, even preliminary analyses reveal that much more information receives focal treatment (i.e. is stressed) than is new or newsworthy information. For further clarification of these processes, this work looks to Mental Spaces theory (Fauconnier 1985/1994).

\subsection{Mental Space Navigations}

Within the Mental Spaces approach, contributions consist of two fundamental elements: spaces and those propositions to be processed within them. Examples of how these two elements are unambiguously separated can easily be found. For example, in (1), truth of the queried proposition is that an acceptable thing is clearly limited to the locative space in Sabah, in Borneo. If the speaker were to have used a different spatial definition (e.g. in Queensland), she would have changed the context in which the proposition can be measured as true or false.

$$
\mathrm{H} \text { : is that an ac'ceptable thing } \uparrow \text { in in in Sabah } \uparrow \text { in Borneo } \uparrow^{2}
$$

This example illustrates that primary stress (shown as bold text) occurs in both in the propositional component and the spatial definition.

In contrast with (1), however, there are cases where the distinction between proposition and space is less obvious. For example, in (2) a number of locative phrases provide additional information to the propositional component, but do not function as the contextual spaces in which the propositions are to be processed. The breakdown of Proposition to Spaces for examples (1) and (2) is given in (3).

\footnotetext{
${ }^{2}$ Examples are given with the following additional information: bold $=$ primary stress (prosodic pulse); underlined $=$ where verbal and gesture components coincide; arrow $\downarrow$ above text $=$ gesture onset; arrows $(\uparrow \downarrow \rightarrow$ within text examples $)=$ intonation contour. Note that an arrow $\rightarrow$ in front of an example indicates the particular line to which attention is being drawn.
} 
June Luchjenbroers

(2) E: I had some.. ah overseas students staying with me $\rightarrow$

(3) Propositional Component + Pointer [line 8] I had overseas students staying [line 9] They'd just come

[line 10] They were in [line 15] Is that an ac'ceptable thing [line 16]

vs. Space Builder with me from Sabah in Borneo foundation year in in in Sabah in Borneo

Examples such as these illustrate that although locative phrases are prime candidates for space builders, they do not always function as such; however, I would argue that their spatial nature is not entirely lost, and in a sense act as "pointers" to potential spaces outside the immediate context of talk (Luchjenbroers 1993). At this point I offer the suggestion that the first mentions in lines 8-10 are references to locations "outside" focus ("F-space"), while the second reference in lines 15-16 becomes "inside" focus, as it here functions as the contextual space in which the new proposition is to be considered. The difference between pointers and spaces is that the latter add structure to the developing discourse network (cf. Sweetser and Fauconnier 1996).

Of interest also is that these locative phrases attract prosodic stress in both examples (1) and (2). This gives evidence that the presumed correlation between new information and stress is not two-way: although what the speaker predicts to be new information to the hearer attracts stress, stress clearly must correlate with more than new/newsworthy information. I offer the suggestion that the repeated stress in the later talk (later by only five main clauses) serves to amplify a shift in function from the propositional component (line 9) to the space in which the new proposition is to be processed (lines 15-16). Hence, prosodic stress signposts that information either (i) is new, whether spatial, pointer, or propositional, or (ii) has changed function, such as movement from proposition to spatial definition. Alternatively, following the suggested terminology above, prosodic stress also signposts that information has moved from outside to inside F-space. Preliminary observations suggest that movement in the other direction (from inside to outside F-space) does not attract prosodic stress; in such cases the general rule of "given information is not focal" is seen to apply.

A final observation is that when an active space is altered in some way, the altered part will likely attract stress, e.g. (4).

(4) E: they 'didn't have much experience in essay writing in $\rightarrow$ (in breath) in the English style $\uparrow$

In (4) the spatial reference in essay writing (line 20) is a first mention and is in focus but does not attract stress; presumably because it is so deeply associated 
with the primary topic (plagiarism) it is considered active. In contrast, the subsequent spatial modification in the English style, which should otherwise be unstressed for the same reason, contrasts with the spatial context of talk before line 20 , practices in Borneo, and is therefore stressed. There is good reason to utilize the known phenomenon of 'contrastive stress' for mental space maneuvers, and this will emerge in later discussions also, but arguably only when information moves into focus.

\subsection{Discourse 'Levels'}

A full consideration of the data has also prompted a consideration of the embeddedness of the spaces referred to in talk. In particular, at least four distinct levels (or layers) have become apparent (see also Rubba 1996). These four levels, illustrated in Figure 1, are defined as follows.

Level 1: the here and now context of talk, which is the dynamic of being in an enclosed area, talking to a fellow student who they do not know well (if at all), in front of a video camera and an academic who is taping every word. This is the most primary space (cf. Base Space), and talk at any time can revert to this layer, e.g. line 349 in (5).

(5) $\rightarrow$ F: gosh I sure like this pen $\downarrow$

$\mathrm{S}$ : I like it too (both giggle)... umm..

F: ok. what about exams now....

Within this general context layer, speakers need to contend with a number of factors in addition to the task being performed. For example, they need to deal with the interpersonal dynamics of the situation, such as social rules of interaction (how to proceed), as well as live up to personal ideals, such as 'look intelligent', and 'don't let the other person get the better of you', etc. Notably, line 351 takes talk back to the next level of talk, Level 2.

Level 2: refers to the Task speakers are involved in. At this level, speakers negotiate how they will proceed, e.g. (6). It is a level of interaction that carves up the subject matter into manageable chunks.

$$
\begin{gathered}
\mathrm{H}: \text { I guess I guess. what we should do is is um.. } \downarrow \\
\rightarrow \quad \text { de'c-ide what plagiarism is first } \rightarrow
\end{gathered}
$$

Dealing with the task involves a range of issues, including defining the problem (which in these data takes the form of recognizing how the problem is manifested), producing strategies for dealing with the problem, and identifying the consequences of being found guilty. The abstract examples of these Level 2 categories make up the next level of talk, Level 3. 
Figure 1. Embedding of Levels

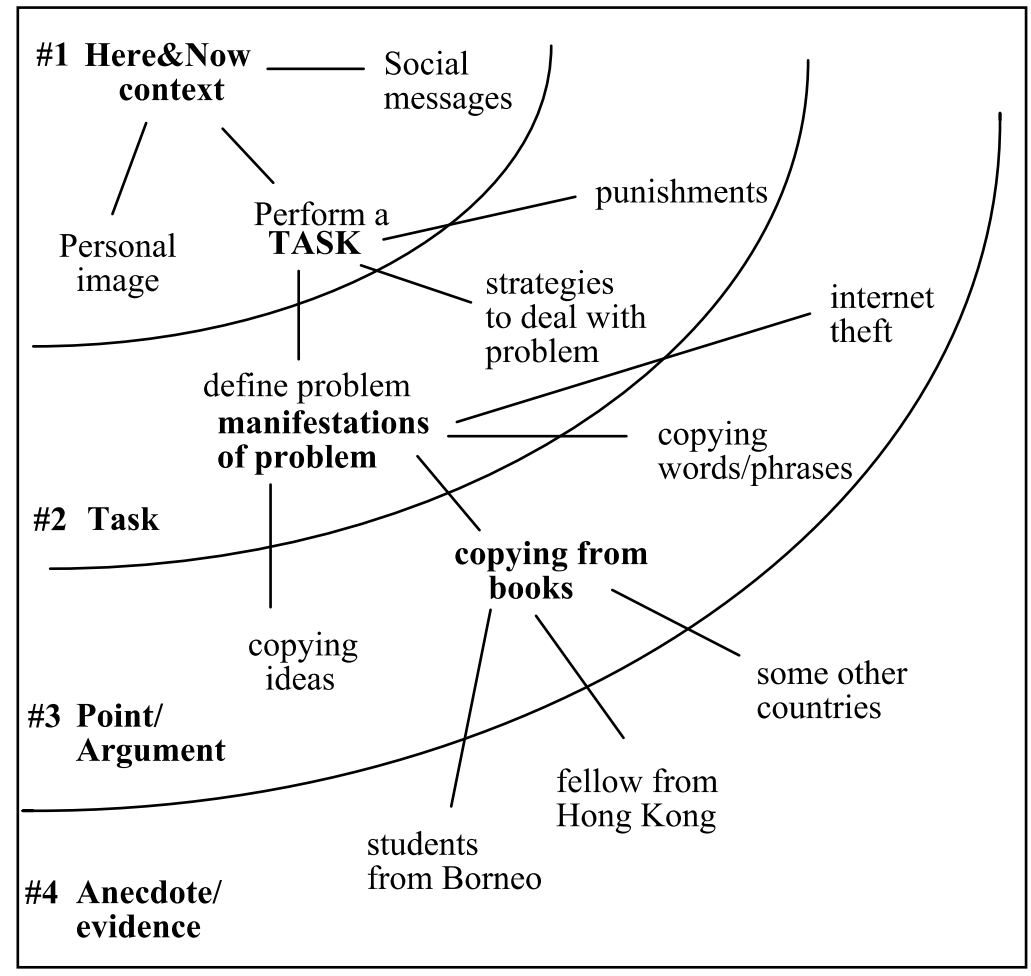

Level 3: includes the Points or Arguments that emerge from dealing with the Task, e.g. (7). These are general statements that define or explain the tasks decided upon in the Task layer.

E: oh it's.. more or less whole. sale copying from books $\rightarrow$

Level 4: is the final level and includes Anecdotal evidence of the Points or Arguments given in talk, e.g. (8).

(8) $\rightarrow$ E: I had some.. ah overseas students staying with me $\rightarrow$ and they used to copy. whole sale. pieces from books $\rightarrow$

Here again, specific evidence defines or explains individual (abstract) Points (Level 3) which combined make up different aspects of the Task (Level 2), and everything to do with the task is a part of what the speaker is dealing with in the here-and-now of talk (Level 1). It may be difficult to imagine that speakers keep track of where they are in the tree at all times; nevertheless, if a hearer were to 
confuse how a piece of evidence proves a Point, or how a Point was relevant to the Task being performed, either miscommunication would follow or comprehension would fail.

Possible evidence for this Levels approach may be provided by accompanying stress. In particular, a Levels analysis may clarify why some (otherwise given) spaces attract stress when a simple approach to either spaces or information flow might predict otherwise, e.g. (9).

$$
\begin{aligned}
& \text { E: oh it's.. more or less whole. sale copying from books } \rightarrow \\
& \quad \text { H.... } \\
& \text { H: so i-i-if we sa-ay. first of all that plagiarism } \rightarrow \\
& \text { 's just whole sale copying of stuff from books an that } \rightarrow
\end{aligned}
$$

In (9), the lexical element of interest is the propositional component (Pointer) from books which is stressed in these two mentions (lines 8 and 27, said roughly 35 seconds apart). The information is not new and in such a short time span is certainly still active; however, movement around the network is complex. The contribution in line 8 functions from the Point Level, and between lines 8 and 27 talk moves from that Level to Evidence \#1, up to the Task Level and then to Evidence \#1 again, followed by Evidence \#2, and then in line 26 back to the Point Level. Prosodic stress continues where the lexical component is given, but the mental space in which it must function changes. I suggest that continued prosodic stress of such active components can be explained as a speaker cue to hearers about how to navigate conceptual and discourse space.

The Levels approach suggested here also complements the inside vs. outside F-space suggestion offered above. In line 27 the element (copying) from books is moved back into focus. Hence this example illustrates how prosodic stress, Levels, and inside focus converge.

Observations of the data so far have led to the following generalizations:

a. New or newsworthy Information (whether as Space or Proposition) will likely attract primary stress.

b. When Propositional information becomes the spatial definition of a subsequent Proposition (i.e. moves from outside to inside F-space), it will likely attract primary stress.

c. When spatial information is modified in some way (i.e. moves into Fspace), the modified part will likely attract primary stress.

d. When information (whether Space or Proposition) signposts a different Level in talk (i.e. a different Level moves into F-space), it will likely attract primary stress.

In these examples I have shown that stress assignment goes beyond new information and is an important cue to hearers about how to maneuver very intricate 
June Luchjenbroers

aspects of discourse structure. In the following section I will consider how gesture may be said to join in.

\section{Gestural Cues}

When speakers gesture, the gestures they produce take place within a comfortable physical space in front of them. The dimension of this space is roughly the shape of a cube that runs from shoulder to waist in height, from elbow (at the waist or, in these data, the table) to the hand in depth, and has body width. The actual size of a speaker's gesture space (cube) varies from speaker to speaker, and very likely from culture to culture, as does the proportion of gesture to speech. In general, speakers who are less animated in gesture use a smaller gestural cube, and those who are more animated use a larger cube that is more consistent with the dimensions given. The size of a speaker's gesture space is defined by where they make most of their gestures. However, in addition to where speakers make most of their gestures they also make numerous gestures that are clearly outside these general boundaries. I suggest that these general vs. extreme boundaries are consistent with inside vs. outside gestural F-space, and when a gesture is made within the cube, or not, the speaker is conveying additional but relevant information about navigating mental space.

To further clarify this suggestion, gestures are considered from a number of angles. In particular, this research has considered gesture in terms of (i) gestural types, (ii) gestural complexity, and (iii) how types and complexity convey information to the hearer about mental space navigations.

\subsection{Gestural Types}

Researchers in gesture (see McNeill 2000) generally recognize at least three types of gesture: Deictic or Indexical gestures, Iconic gestures, and Pantomimes. In this paper I will discuss Indexical and Iconic gestures. Indexicals are the most basic form of gesture and refer specifically to gestures involving (a) only the index finger, e.g. (10), (b) the full hand, e.g. (11), or (c) an instrument such as a pen, e.g. (12).

(10) E: there's even a special section of legal studies at $\underline{Q U T}$ $\mathrm{L}$ arm crosses the body ( $\&$ F-space)

$\mathrm{L}$ finger points away from $\mathrm{S}$ in direction of QUT ( $\neq \mathrm{F}$-space)

(11) H: but... of course at the university that's. that's not on $\uparrow$ both hands in a flat cup, palms down \& slanting inwards, fingers touching the table, in front of S (= F-space)

(12) E: they say the university policy here .. is pen points down, touching the table, in front of S (= F-space)

In all three examples the physical location of the referent has a direct relation to the physical location pointed to by the speaker. Also, in terms of F-space, the 
relation between the physical locations of the referents and the indexical gestures is no coincidence. The gestures given in examples (11) and (12), referring to "here", are firmly in the center of the speaker's gestural space (i.e. in F-space), but example (10), referring to a different university, is clearly outside it. This gestural choice is not arbitrary; the speaker could have chosen the right hand to make this gesture to the right (when writing the speaker is also right-handed), in which case the indexical would still have been outside F-space but that choice might have been coincidental and therefore ambiguous. In this case the speaker's choice to use the left hand to cross the body (and F-space) to a position that is again outside the speaker's gestural F-space is more telling of the speaker's intent and the focal status of that information. The body of talk is about practices at university (in Australia), but the gestures make clear that for these speakers, the specific space that is maximally active (and focal) is what happens "here" (at the University of Queensland) as opposed to "not here".

Notably, all three examples are also examples of contrastive stress. In section 2 of this paper, stress was associated with bringing elements into focus, either because they convey new information or because they involve processing at a different conceptual level from the contribution before. Examples (10)-(12) reveal a similar process in that in these examples stress amplifies a change in spatial definition to spaces that are either new or renewed. However, these (re-) activated conceptual spaces also deal with locations in physical space, and the gesture placements either inside or outside F-space amplify the relation of those locations to the speaker. Hence, Indexical gestures go beyond lexical instructions for mental space creation and identification.

Similarly, Iconic gestures can function like Indexicals (i.e. point to physical locations), although the relation between the gesture and the referent is not always straightforward. Iconic gestures often convey information that is relevant to more than one domain in mental space navigations, e.g. (13a).

(13) a. H: that's... completely peppered with.. you know tht tt t

$\mathrm{R}$ hand, pinched (all four fingers on top of thumb), pointing to a number of points very close together, above $\mathrm{R}$ eye ( $\neq \mathrm{F}$-space)

b. In this hypothetical student paper, there are many plagiarized bits.

In (13a) the speaker points to locations in physical space (above F-space). The Iconic gestures represent the unseen pages of a typical student paper that has been plagiarized, and the points in space refer to locations in that hypothetical paper. The frequency of the jabbing motions to locations in the air correlates with the frequency of plagiarized sections in that student paper. The gestures are clearly locational but the information is part of the propositional component within the space that is created by virtue of the gesture. An extended interpretation of this sequence would be a verbalized string such as given in (13b). The space builder in this hypothetical student paper is created to make sense of the gesture, and the proposition there are many plagiarized bits is conveyed by the high number of 
June Luchjenbroers

jabs in space, and serves as an elaboration of the proposition already verbally conveyed. Hence the gesture conveys both spatial and propositional information.

Another important feature of Iconic gestures that appear to have an Indexical character is that they may be relevant to recognizing the Levels (of embeddedness) in talk referred to earlier, e.g. (14) and (15).

(14) H: so i-i-if we sa-ay. first of all that plagiarism $\rightarrow$

$\rightarrow \quad$ 's just whole sale copying of stuff from books an that $\rightarrow$

$\mathrm{L}$ hand serves as a list, $\mathrm{R}$ hand points to fingers, each referring

to a different Point for the Task (define the problem) (= F-space)

\footnotetext{
$\mathrm{H}$ : even even copying someone else's assignment index finger pointing at $\mathrm{H}(=\mathrm{F}$-space $)$
}

Examples like (14) and (15) involve pointing gestures that do not refer to the content at all. These are interactional because they serve as a kind of instruction to the hearer. In (14), the gesture instructs the hearer that the speaker's contribution is a Point to be added to the list of Points. Similarly, in (15), the pointing finger is not an accusation that the hearer is guilty of the said act, but here the gesture also functions as an instruction to the hearer to add a new Point to the list of Points she is jotting down. In cases such as these, gestures instruct hearers to shift Levels; they are not for clarifying or complementing content.

It is clear from examples like (13)-(15) that gestures can convey different kinds of information to the lexical component. Similarly, in (16) it is difficult to categorize the associated gesture as spatial or propositional.

$$
\text { H: so we 'say if you do.. this.. and this and this }
$$

$\mathrm{R}$ hand, pinched (all four fingers on top of thumb), pointing to a number of equidistant points in space, forming an oblique row from just above F-space (height of L eye), into F-space (below $R$ shoulder)

In (16) the speaker uses pointing gestures to possible events (that constitute plagiarism) as locations in physical space. In this example the relationship between gesture icon and referent is more abstract than any of the earlier examples as the gestures refer to possible events and not locations. In examples (14) and (15) the location is arguably either the list that the speaker has made with her fingers, or the list of Points (Arguments) the other person is writing down. However, the pointing gestures in (16) refer to Points made earlier in talk, and which can reasonably be expected to exist in conceptual structure. These pointing gestures therefore are locational but in a more abstract sense than the earlier examples. 
The complexity of this example is greater than deciding whether to analyze the gesture as spatial, pointer, or proposition, as it also refers the speaker back to another Level of talk: the Task Level (having just discussed more evidence). The Points/Arguments in conceptual space, indicated by this.. and this and this, were all produced with prosodic stress, which would be expected when Levels jump back into focus, as well as when contrasting discourse elements are specified. Nevertheless, the conceptual load in processing all informational cues is enormous, the complexity of which is missed by just an analysis of the lexical component.

\subsection{Gestural Complexity}

As already evident in the examples above, gestures can very complex. The data also contains many 'simple' gestures (i.e. those that convey a straightforward semantic relationship between the essential message carried by the gesture and the lexical component it accompanies). For example, a frequent simple gesture used during these talks is a take gesture (one hand scoops an unseen substance or object and draws it to the body), which was used to complement talk of taking, stealing, plagiarizing, and cheating. Such gestures are simple because they are consistent with the verbal component. However, examples where gestures point to iconic locations, such as in (13) above, or events, such as in (16) above, are complex because (among other things) the physical location of the gestures holds no straightforward relation to the referents. In the latter cases, gestures do substantially more than clarify lexical meanings; they complement the lexical component by providing meanings the speaker does not (need to) articulate.

Complex gestures also vary in the range of complexity involved, from the very complex examples already given to less complex examples where the gesture meaning is closely related to the meaning conveyed lexically, e.g. (17).

$$
\begin{aligned}
& \text { H: like-t.. it's like, it's some sort of public.. public information } \\
& \text { both hands splayed, palms facing each other, making circles } \\
& \text { opposite each other (= F-space) }
\end{aligned}
$$

In (17) the gesture associated with public suggests "mixing", which conveys an attribute that the word does not entirely convey. Here the sense that is added is that public information is a mixture from multiple sources (who cares where?), and it is therefore legal to mix that information with one's own resources. Example (18) below also utilizes the "mixing" gesture, but is more complex because it also conveys additional information through the use of F-space.

(18) E: so.. he just couldn't.. turn that information around.. both hands, palms facing $\mathrm{S}$, rotating from away from $\mathrm{S}$, up and over the other hand to closer to $\mathrm{S}$, several times (= mixing outside F-space into F-space) 
June Luchjenbroers

Example (18) involves a component of meaning that is not only clarifying of the lexical component, such as in (17), but is also complementary. The alternating positioning of the hands correlates with the lexical component turn around but the gesture conveys scooping an invisible substance toward the speaker in a large circular motion and involves moving the hands from outside F-space into F-space. The directionality of this gesture is significant because the resting state for each hand is roughly the center of the chest, which clearly conveys "mine" (or, in this case, "make mine"). This is a clear example of how the boundaries of F-space can be used to complement both the semantics of the lexical component as well as the gesture. This sense extension relies on the contrast between inside F-space $=$ "here" and "me/mine" vs. outside F-space = "not here" and "not me/mine".

In sum, gestural complexity involves multiple types of information: spatial, propositional, and sometimes interactional. Unlike the lexical component which can generally be unambiguously assigned one or other mental space role (i.e. space builder or proposition), gestures often contain components with multiple roles. The issue remaining is to determine how gestural and prosodic features may be said to combine to disambiguate the complexity of the hearer's input.

\subsection{Prosody and Gesture: Mental Spaces Cues}

Mental spaces require spatial definitions in which propositions can be processed. However, the kinds of information both prosodic and gestural information provide has already been shown to advance on these two elements, with some clear correlates with Levels of embeddedness, as well as gestures that appear to convey both spatial and propositional information. In addition, gestures sometimes provide interactional information to the hearer. In examples (14) and (15) above, these were instructions relating to contextual Levels, but other examples reveal how gestures combine with prosodic information to achieve the same interactional outcome, e.g. (19), where the gesture together with an increase in stress and slowing of speech indicates that the speaker has completed her turn and is offering up the floor.

$$
\begin{aligned}
& \text { E: } n \text { also there's a fellow there from Hong Kong you know... } \\
& \quad \text { he was reeal-ly ba-ad.. } \\
& \text { nodding head (= end of turn) }
\end{aligned}
$$

In contrast, however, other examples have revealed how prosodic stress and gestural information appear to convey very different kinds of information. In the Indexical gesture examples (10)-(12), prosodic stress amplified a change in spatial definition while the gestural placements, either inside or outside F-space, amplified how those locations relate to the speaker. Essentially the gestural and prosodic cues in these examples provided very different kinds of information that serve to offer greater depth to the information offered in the speech stream.

The range of examples presented provide a number of meanings for F-space. In terms of prosodic stress, focus is related to speaker assumptions about what would be new information to the hearer, and to changes in the location in which 
Cues for Navigations around Mental Space

an utterance operates within the mental spaces network, which in some cases is represented as Levels of embeddedness. In terms of gesture space, however, focus refers to either the micro-context in which a proposition is to be processed (akin to space builders or the propositions within them), or to another important form of contrast, that between aspects more closely associated with the individual ("here", "me", and "mine") vs. aspects that are not ("not here", "not me", and "not mine").

In addition to this egocentric nature of a person's gesture space, however, gestures often convey important information about spatial definitions and also often amplify changes in them, e.g. (20).

\section{(20) H:I 'think... I 'think sn 'some other countries} hands together (prayer posture) in front of $\mathrm{S}$ (= F-space)

$\rightarrow \quad$ well not just other countries but I I 'think some 'people um. hands move apart from prayer posture, in front of S (= F-space)

After a space has been created, an alteration of that space will involve a change in hand gesture. In (20), line 34 is said with both hands together, which does not indicate anything about the space (other than maybe illustrating the speaker's contemplation), but the change in space in line 35 motivates the speaker to move her hands apart. This is not a coincidence; the space has been broadened (widened) to include more people and the space between the hands has likewise been increased. The hearer is also alerted by the stress on just which coincides with the change in gesture position.

\section{Is This a Concert?}

In this paper I have presented a number of examples to suggest that the answer to this question is a tentative "yes". I make this suggestion tentatively because although there are many cases where the informational load of gestural information substantially enriches the speech stream to which a hearer is exposed, there are also numerous cases where gestural information substantially precedes the lexical component, in which case there is reason to suppose that gestures might often be indicative of speakers making their own way through mental space, as they are hearer-oriented cues to facilitate comprehension during discourse.

There is also clear evidence of a division of labor between prosodic and gestural information, which often converge and complement each other by enriching the information potentially available to the hearer. Stress assignment is clearly related to presumed accessibility of the spatial component of an utterance and therefore plays a role in informing hearers about navigating space in terms of how far they need to search (or how far the speaker wants/expects them to search). In terms of gesture, however, the boundaries between space and proposition are sometimes blurred as multiple types of information can simultaneously be conveyed. In particular, gestures may clarify an element that belongs within the propositional element, but may be produced in a physical space that lies outside gestural F-space; therefore, they might convey spatial information about the 
June Luchjenbroers

location. Similarly, in some cases, gestures convey information about what level that information pertains to (e.g. examples or task). In sum, the complexity of gestures is scarcely understood, and much more data needs to be analyzed to alter that state. It is, however, clear from the examples presented in this paper that the role of gestures (together with prosodic information) is a fruitful and important area of investigation for a Mental Spaces approach to discourse.

\section{References}

Chafe, Wallace. 1994. Discourse, Consciousness, and Time. Chicago: University of Chicago Press.

Fauconnier, Gilles. 1985/1994. Mental Spaces: Aspects of Meaning Construction in Natural Language. New York: Cambridge University Press.

Lambrecht, Knut. 1994. Information Structure and Sentence Form: Topic, Focus and the Mental Representations of Discourse Referents. Cambridge: Cambridge University Press.

Luchjenbroers, June. 1993. Pragmatic Inference in Language Processing. Ph.D. diss., La Trobe University, Melbourne, Australia.

Luchjenbroers, June. In press. "Functionalism, Conceptual Mappings and Mutual Ground Construction in Court." In P. Casanovas (ed.).

Luchjenbroers, June. In progress. "Conceptual Mappings and Mutual Ground Construction." In A. Verhagen and J. v.d. Weider (eds.), Levels in Cognition.

McNeill, David, ed. 2000. Language and Gesture. Language, Culture, and Cognition. Cambridge: Cambridge University Press.

Rubba, J. 1996. "Interpretation of Deictic Expressions." In G. Fauconnier and E. Sweetser (eds.), Spaces, Worlds and Grammar. Chicago: University of Chicago Press.

Sweetser, E. and G. Fauconnier. 1996. "Cognitive Links and Domains: Basic Aspects of Mental Spaces Theory.” In G. Fauconnier and E. Sweetser (eds.), Spaces, Worlds and Grammar. Chicago: University of Chicago Press.

Department of Linguistics

University of Wales at Bangor

Gwynedd, LL57 2DG, Wales

United Kingdom

junel@bangor.ac.uk 